\title{
Activation and 3D Imaging of Phase-change Nanodroplet Contrast Agents with a 2D Ultrasound Probe
}

\author{
Sevan Harput ${ }^{1,2}$, Ge Zhang ${ }^{2}$, Matthieu Toulemonde ${ }^{2}$, Jiaqi Zhu ${ }^{2}$, Kirsten Christensen-Jeffries ${ }^{3}$, \\ Jemma Brown ${ }^{3}$, Robert J. Eckersley ${ }^{3}$, Chris Dunsby ${ }^{4}$, and Meng-Xing Tang ${ }^{2}$ \\ ${ }^{1}$ Division of Electrical and Electronic Engineering, London South Bank University, London, SE1 0AA, UK \\ ${ }^{2}$ ULIS Group, Department of Bioengineering, Imperial College London, London, SW7 2BP, UK \\ ${ }^{3}$ Biomedical Engineering Department, Division of Imaging Sciences, King's College London, SE1 7EH, London, UK \\ ${ }^{4}$ Department of Physics and the Centre for Pathology, Imperial College London, London, SW7 2AZ, UK \\ E-mail: S.Harput@1sbu.ac.uk, Mengxing.Tang@imperial.ac.uk
}

\begin{abstract}
Nanodroplets are on-demand ultrasound contrast agents. They can remain in circulation longer in vivo than microbubbles and can be spatiotemporally and selectively activated to provide contrast when required. Perfluorocarbon nanodroplets have been used for conventional 2D ultrasound imaging and different types of nanodroplets have been studied and characterized by researchers regarding their suitability for imaging. However, the use of nanodroplets for 3D imaging and their activation using a 2D ultrasound probe has not been reported. In this study, we investigate the nanodroplet activation using a $2 D$ ultrasound imaging probe within clinical safety limits.
\end{abstract}

\section{INTRODUCTION}

Super-resolution ultrasound (SR-US) imaging techniques are capable of breaking the diffraction limit and imaging microvasculature. Localization-based SR-US imaging relies on detection of ultrasound contrast agents' positions with high precision. Locations of spatially isolated point sources from multiple frames are combined to generate a super-resolved image. Most of the existing super-resolution methods use microbubbles [1]-[17]. Microbubbles follow the slow physiological flow inside microvessels and therefore super-resolution requires long data acquisition time, which together with the motion during data acquisition make it challenging for clinical use [10], [18], [19]. The choice of microbubble concentration can also affect the quality of the SR-US image. Multiple overlapping microbubble echoes within the resolution limit can cause position errors, therefore isolated microbubble echoes should be localized with the best possible method [11], [20][23].

Nanodroplets, also called phase-change contrast agents, have been widely investigated for ultrasound imaging [24][26]. They offer potential solutions to aforementioned issues of SR-US imaging with microbubbles. Nanodroplets can be selectively activated on demand to provide an ultrasound contrast signal and can be imaged at high frame rates. Low-boilingpoint nanodroplets have been demonstrated for reducing the SR-US acquisition time as they can be activated, destroyed and imaged on a sub-second time scale [27]. Thanks to these advantages, the use of nanodroplets as an echogenic point source for super-resolution imaging is increasing [28]-[30].

Researchers demonstrated the use of nanodroplets for $2 \mathrm{D}$ ultrasound imaging, including 2D SR-US imaging. However, the use of nanodroplets for 3D ultrasound imaging has not been demonstrated yet. In this study, we investigate the activation process of nanodroplets using a $2 \mathrm{D}$ ultrasound imaging probe within clinical safety limits. The main purpose of this study is to investigate the data acquisition time for nanodropletbased 3D SR-US imaging and minimize the acquisition time by tuning the activation parameters. A range of activation waveforms with varying acoustic pressures are employed to investigate the activation efficiency of nanodroplets. Feasibility of activation and 3D ultrasound imaging of nanodroplets is demonstrated using high frame rate imaging.

\section{Materials AND Methods}

\section{A. Nanodroplet Preparation}

Nanodroplets were fabricated with a Definity-like lipid shell and decafluorobutane (DFB) core with as previously described in [24], [26]. Briefly, a lipid colloid was generated by dissolving a 9:1 molar ratio of 1,2-dipalmitoyl-sn-glycero3-phosphocholine (DPPC) and 1,2-distearoyl-sn-glycero-3phosphoethanolamine-N-[amino(polyethylene glycol)-2000] (DSPE-PEG-2000) into a solution of phosphate-buffered saline (PBS), propylene glycol, and glycerol (16:3:1, V: V) to achieve a total lipid concentration of $1 \mathrm{mg} / \mathrm{mL}$. All the lipids described above were purchased from Avanti Polar Lipids, Inc., USA. A volume of $1 \mathrm{~mL}$ of lipid solution was added to a $2 \mathrm{~mL}$ glass vial. The headspace of the vial was filled with decafluorobutane gas (Fluoromed, USA) via an inlet needle along with a vent needle. Mechanical agitation was applied using a bubble shaker (Vialmix, USA) to produce the stable and microscale bubbles composed of a gaseous decafluorobutane core encapsulated by a lipid shell.

In order to condense microbubbles into nanodroplets, the headspace of the vial was pressurized according to the previously described methods. The vial of microbubbles was 


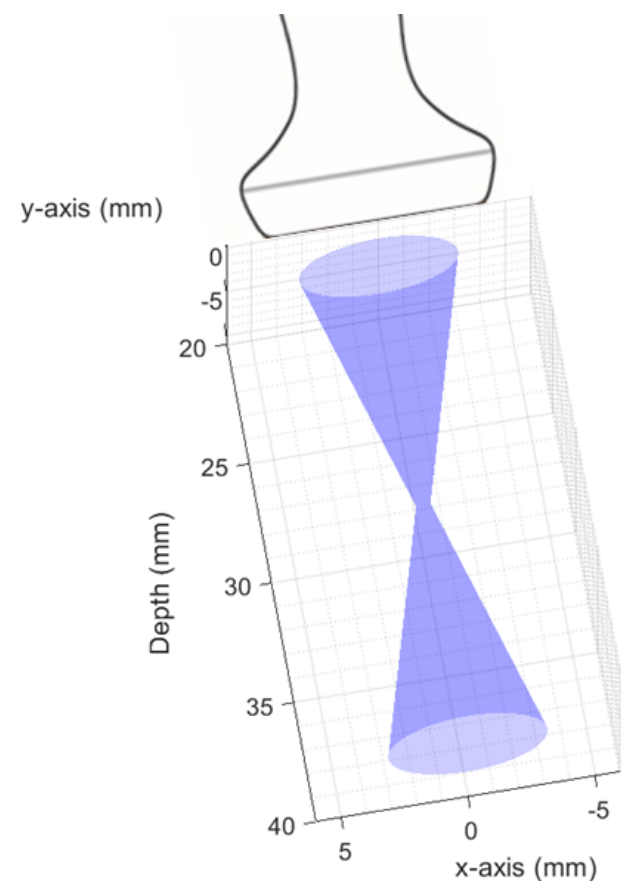

Fig. 1. Illustration of the activation beam profile. Activation beam is focused at $30 \mathrm{~mm}$ depth, but it appears slightly closer to the probe due to the viewing angle in the figure.

immersed in an ice-salt bath $\left(-5\right.$ to $\left.-8^{\circ} \mathrm{C}\right)$ followed by pressurization with ambient air into the vial septum while the vial contents remained in the ice-salt bath.

Size and concentration of the generated nanodroplets were measured by nanoparticle tracking analysis (NTA) using a NanoSight NS300 (Malvern Instruments Ltd, UK). Nanodroplets has a mean diameter of $119 \pm 7 \mathrm{~nm}$ and a mean concentration of $3.5 \times 10^{10}$ nanodroplets $/ \mathrm{mL}$. For the ultrasound experiments, nanodroplets were diluted at a volumevolume ratio of 1:30000 (Native nanodroplet solution: Water) in a beaker at $37^{\circ} \mathrm{C}$.

\section{B. Experimental Setup}

A 512-element 2D sparse array was used for nanodroplet activation and imaging with a center frequency of $3.7 \mathrm{MHz}$ and a bandwidth of 60\%. Two synchronized ULA-OP 256 systems (MSD Lab, University of Florence, Italy) were used to activate nanodroplets and acquire data using the $2 \mathrm{D}$ ultrasound probe [31], [32].

Nanodroplet activation was performed using focused beams with 2, 4, 6 and 10 cycle $4 \mathrm{MHz}$ sinusoidal waveforms focused at a depth of $30 \mathrm{~mm}$, as illustrated in Figure 1. Activated nanodroplets were imaged in $3 \mathrm{D}$ at a center frequency of 4 $\mathrm{MHz}$ using plane waves. After every activation pulse, 100 imaging frames were acquired at an MI of 0.1 and frame rate of $1000 \mathrm{~Hz}$.

Experiments were repeated 5 times for a range of activation pulses with varying amplitudes between $40 \%-100 \%$, where $100 \%$ corresponds to the maximum excitation voltage of 40 Volts, with the MI values given in Table I.
TABLE I

Mechanical Index (MI) OF the Activation Pulse

\begin{tabular}{lcccc}
\hline \hline Amplitude & 2 cycle & 4 cycle & 6 cycle & 10 cycle \\
\hline $40 \%$ Amplitude & 0.75 & 0.84 & 0.88 & 0.90 \\
$60 \%$ Amplitude & 1.05 & 1.10 & 1.20 & 1.22 \\
$80 \%$ Amplitude & 1.35 & 1.48 & 1.55 & 1.58 \\
$100 \%$ Amplitude & 1.65 & 1.81 & 1.90 & 1.90 \\
\hline \hline
\end{tabular}

\section{RESUlTS \& DisCUSSION}

Activated nanodroplets, which are microbubbles, were imaged using the $2 \mathrm{D}$ sparse array probe. Figure 2 shows the $3 \mathrm{D}$ images $(-20 \mathrm{~dB}$ isosurfaces) of the activated nanodroplets for an MI range of 0.75 (40\% amplitude) to 1.65 (100\% amplitude) using a 2 cycle wave. The same experiments were also performed for 4,6 and 8 cycle sinusoidal waves (not shown here). For all activation pulses, the experiment performed at a higher pressure resulted in the activation of more nanodroplets. The results show that increasing the activation MI increased the ultrasound contrast generated by nanodroplets in and around the focal region.

The contrast enhancement was measured around the activation region within a volume of $10 \times 10 \times 10 \mathrm{~mm}^{3}$. The measured average intensity values in this region for different activation amplitudes and waveforms are plotted in Figure 3. All activations generated higher intensity values than the reference experiments shown in Figure 3 (left) without any nanodroplets. The activation amplitude, which changed between an MI of 0.75 and 1.9 as given in Table I, had a positive correlation with image intensity. Increasing the number of cycles in the activation waveform also increased the image intensity.

This study combined the recent advances in high-frame-rate 3D ultrasound imaging and ultrasound contrast agents with a motivation of reducing the imaging acquisition duration for 3D super-resolution ultrasound imaging. The achieved results in this study agree with the previous research performed using 2D ultrasound imaging. Changing the number of cycles and acoustic pressure of the activation pulses affects the image contrast, which is proportional to nanodroplet activation.

\section{CONCLUSION}

This study presents an in vitro demonstration of nanodroplet activation using 3D ultrasound imaging. Results show that increasing the number of transmit cycles and acoustic pressure can increase the number of activations, which was measured as a change in the image contrast over the imaging volume. The activation of nanodroplets can be controlled by tuning the activation pulses for specific applications. Controlling the nanodroplet activation events may be important in droplet-based super-resolution imaging for minimizing the data acquisition time.

The advantage of using 3D imaging and activation is that the experiments can be performed without confining the nanodroplets. By measuring all activation events in the imaging 

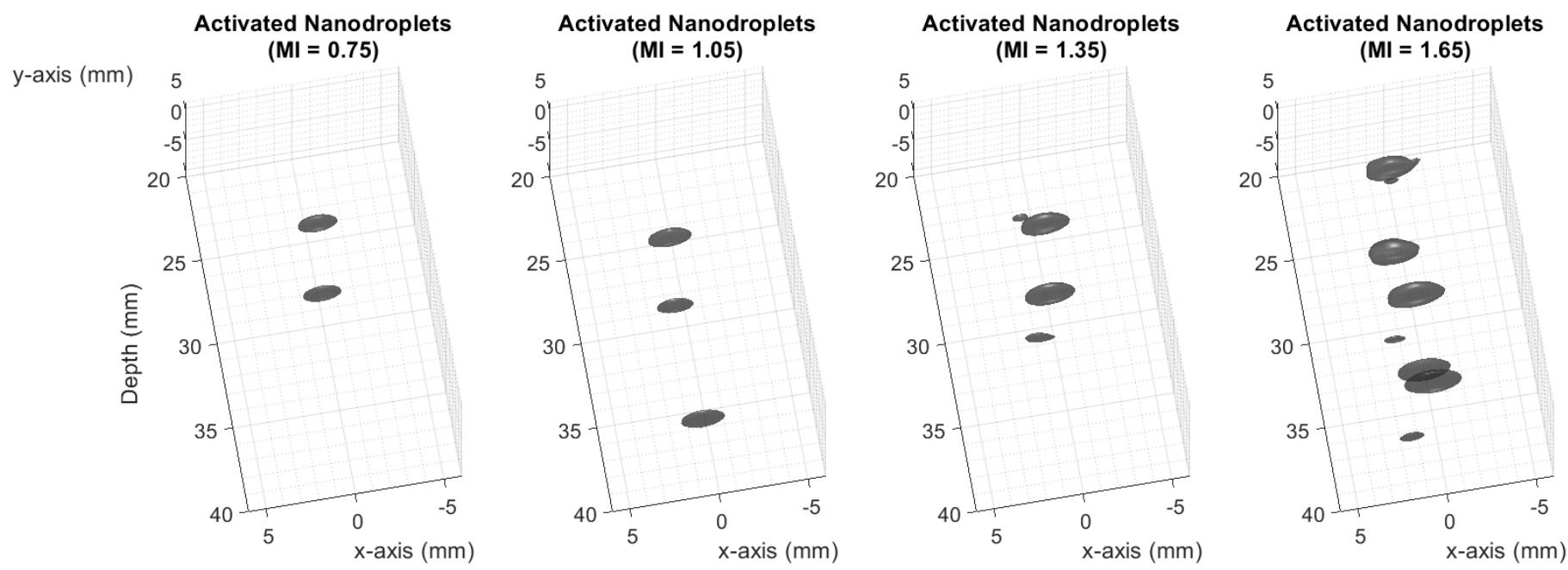

Fig. 2. 3D image of the activated nanodroplets using a 2 cycle sinusoidal excitation waveform focused at $30 \mathrm{~mm}$ (shown in Figure 1 ) with a varying MI between 0.75 and 1.65 .
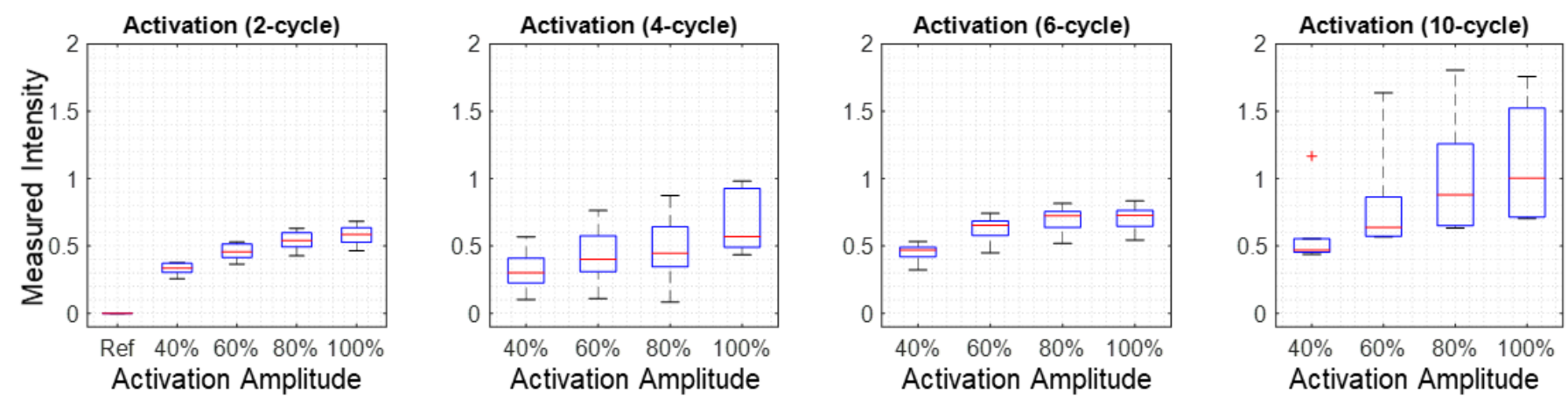

Fig. 3. Peak intensity of nanodroplets measured at the focal region of the activation beam for different excitation pressures and number of cycles. Results are normalized to the maximum mean intensity. See Table I for the corresponding MI values.

volume, signals are less likely to be lost due to out of plane motion in 2D imaging.

\section{ACKNOWLEDGMENTS}

This work was supported mainly by the EPSRC under Grant EP/N015487/1 and EP/N014855/1, in part by the King's College London (KCL) and Imperial College London EPSRC Centre for Doctoral Training in Medical Imaging (EP/L015226/1), in part by the Wellcome EPSRC Centre for Medical Engineering at KCL (WT 203148/Z/16/Z), in part by the Department of Health through the National Institute for Health Research comprehensive Biomedical Research Center Award to Guy's and St Thomas' NHS Foundation Trust in partnership with KCL and King's College Hospital NHS Foundation Trust, in part by the Graham-Dixon Foundation and in part by NVIDIA GPU grant.

\section{REFERENCES}

[1] O. M. Viessmann, R. J. Eckersley, K. Christensen-Jeffries, M.-X. Tang, and C. Dunsby, "Acoustic super-resolution with ultrasound and microbubbles," Phys. Med. Biol, vol. 58, pp. 6447-6458, 2013.

[2] M. A. O'Reilly and K. Hynynen, "A super-resolution ultrasound method for brain vascular mapping," Medical Physics, vol. 40, no. 110701, 2013.
[3] Y. Desailly, O. Couture, M. Fink, and M. Tanter, "Sono-activated ultrasound localization microscopy," Applied Physics Letters, vol. 103, no. $174107,2013$.

[4] K. Christensen-Jeffries, R. J. Browning, M.-X. Tang, C. Dunsby, and R. J. Eckersley, "In vivo acoustic super-resolution and super-resolved velocity mapping using microbubbles," IEEE Trans. Med. Imag., vol. 34 no. 2, pp. 433-440, 2015.

[5] Y. Desailly, J. Pierre, O. Couture, and M. Tanter, "Resolution limits of ultrafast ultrasound localization microscopy," Phys. Med. Biol, vol. 60, pp. 8723-8740, 2015.

[6] C. Errico, J. Pierre, S. Pezet, Y. Desailly, Z. Lenkei, O. Couture, and M. Tanter, "Ultrafast ultrasound localization microscopy for deep superresolution vascular imaging," Nature, vol. 527, pp. 499-507, 2015.

[7] D. Ackermann and G. Schmitz, "Detection and tracking of multiple microbubbles in ultrasound b-mode images," IEEE Trans. Ultrason., Ferroelectr., Freq. Control, vol. 63, no. 1, pp. 72-82, 2016.

[8] J. Foiret, H. Zhang, T. Ilovitsh, L. Mahakian, S. Tam, and K. W. Ferrara, "Ultrasound localization microscopy to image and assess microvasculature in a rat kidney," Scientific Reports, vol. 7, no. 13662, pp. 1-12, 2017.

[9] F. Lin, S. E. Shelton, D. Espindola, J. D. Rojas, G. Pinton, and P. A. Dayton, "3-d ultrasound localization microscopy for identifying microvascular morphology features of tumor angiogenesis at a resolution beyond the diffraction limit of conventional ultrasound," Theranostics, vol. 7, no. 1, pp. 196-204, 2017.

[10] S. Harput, K. Christensen-Jeffries, J. Brown, Y. Li, K. J. Williams, A. H. Davies, R. J. Eckersley, C. Dunsby, and M. Tang, "Two-stage motion correction for super-resolution ultrasound imaging in human 
lower limb," IEEE Trans. Ultrason., Ferroelectr., Freq. Control, vol. 65, no. 5, pp. 803-814, 2018.

[11] P. Song, J. D. Trzasko, A. Manduca, R. Huang, R. Kadirvel, D. F. Kallmes, and S. Chen, "Improved super-resolution ultrasound microvessel imaging with spatiotemporal nonlocal means filtering and bipartite graph-based microbubble tracking," IEEE Trans. Ultrason., Ferroelectr. Freq. Control, vol. 65, no. 2, pp. 149-167, 2018.

[12] T. Opacic, S. Dencks, B. Theek, M. Piepenbrock, D. Ackermann, A. Rix, T. Lammers, E. Stickeler, S. Delorme, G. Schmitz, and F. Kiessling, "Motion model ultrasound localization microscopy for preclinical and clinical multiparametric tumor characterization," Nature Communications, vol. 9, no. 1527, pp. 1-13, 2018.

[13] T. Ilovitsh, A. Ilovitsh, J. Foiret, B. Z. Fite, and K. W. Ferrara, "Acoustical structured illumination for super-resolution ultrasound imaging," Communications Biology, vol. 1, no. 3, 2018.

[14] S. Harput, K. Christensen-Jeffries, J. Brown, J. Zhu, G. Zhang, C. H. Leow, M. Toulemonde, A. Ramalli, E. Boni, P. Tortoli, R. J. Eckersley, C. Dunsby, and M.-X. Tang, "3-d super-resolution ultrasound imaging using a 2-d sparse array with high volumetric imaging rate," in IEEE International Ultrasonics Symposium (IUS), 2018, pp. 1-4.

[15] J. Zhu, E. M. Rowland, S. Harput, K. Riemer, C. H. Leow, B. Clark, K. Cox, A. Lim, K. Christensen-Jeffries, G. Zhang, J. Brown, C. Dunsby, R. J. Eckersley, P. D. Weinberg, and M.-X. Tang, "3d super-resolution us imaging of rabbit lymph node vasculature in vivo by using microbubbles," Radiology, vol. 291, no. 3, pp. 642-650, 2019, pMID: 30990382.

[16] B. Heiles, M. Correia, V. Hingot, M. Pernot, J. Provost, M. Tanter, and O. Couture, "Ultrafast $3 \mathrm{~d}$ ultrasound localization microscopy using a 3232 matrix array," IEEE Trans. Med. Imag., vol. 38, no. 9, pp. 20052015, 2019.

[17] S. Harput, K. Christensen-Jeffries, A. Ramalli, J. Brown, J. Zhu, G. Zhang, C. H. Leow, M. Toulemonde, E. Boni, P. Tortoli, R. J. Eckersley, C. Dunsby, and M.-X. Tang, "3-d super-resolution ultrasound imaging with a 2-d sparse array," IEEE Trans. Ultrason., Ferroelectr., Freq. Control, in press.

[18] S. Harput, K. Christensen-Jeffries, Y. Li, J. Brown, R. J. Eckersley, C. Dunsby, and M.-X. Tang, "Two stage sub-wavelength motion correction in human microvasculature for ceus imaging," in IEEE International Ultrasonics Symposium (IUS), 2017, pp. 1-4.

[19] S. Harput, K. Christensen-Jeffries, J. Brown, J. Zhu, G. Zhang, R. J. Eckersley, C. Dunsby, and M.-X. Tang, "3-d motion correction for volumetric super-resolution ultrasound imaging," in IEEE International Ultrasonics Symposium (IUS), 2018, pp. 1-4.

[20] K. Christensen-Jeffries, S. Harput, J. Brown, P. N. T. Wells, P. Aljabar, C. Dunsby, M.-X. Tang, and R. J. Eckersley, "Microbubble axial localization errors in ultrasonic super-resolution imaging," IEEE Trans. Ultrason., Ferroelectr., Freq. Control, vol. 64, no. 11, pp. 1644-1654, 2017.

[21] S. Harput, K. Christensen-Jeffries, J. Brown, R. J. Eckersley, C. Dunsby, and M.-X. Tang, "Localisation of multiple non-isolated microbubbles with frequency decomposition in super-resolution imaging," in IEEE International Ultrasonics Symposium (IUS), 2017, pp. 1-4.
[22] P. Song, A. Manduca, J. D. Trzasko, R. E. Daigle, and S. Chen, "On the effects of spatial sampling quantization in super-resolution ultrasound microvessel imaging," IEEE Trans. Ultrason., Ferroelectr., Freq. Control, vol. 65, no. 12, pp. 2264-2276, 2018.

[23] S. Harput, L. H. Fong, A. Stanziola, G. Zhang, M. Toulemonde, J. Zhu, K. Christensen-Jeffries, J. Brown, R. J. Eckersley, E. Grisan, C. Dunsby, and M.-X. Tang, "Super-resolution ultrasound image filtering with machine-learning to reduce the localization error," in IEEE International Ultrasonics Symposium (IUS), 2019, pp. 1-4.

[24] T. Matsunaga, P. Sheeran, S. Luois, J. Streeter, L. Mullin, B. Banerjee, and P. Dayton, "Phase-change nanoparticles using highly volatile perfluorocarbons: toward a platform for extravascular ultrasound imaging," Theranostics, vol. 2, no. 12, pp. 1185 - 1198, 2012.

[25] P. S. Sheeran, J. D. Rojas, C. Puett, J. Hjelmquist, C. B. Arena, and P. A. Dayton, "Contrast-enhanced ultrasound imaging and invivo circulatory kinetics with low-boiling-point nanoscale phase-change perfluorocarbon agents," Ultrasound in Medicine \& Biology, vol. 41, no. 3, pp. 814 831, 2015.

[26] S. Lin, G. Zhang, A. Jamburidze, M. Chee, C. Leow, V. Garbin, and M. Tang, "Imaging of vaporised sub-micron phase change contrast agents with high frame rate ultrasound and optics," Phys. Med. Biol, vol. 63 , no. 6, p. $065002,2018$.

[27] G. Zhang, S. Harput, H. Hu, K. Christensen-Jeffries, J. Zhu, J. Brown, C. H. Leow, R. J. Eckersley, C. Dunsby, and M.-X. Tang, "Fast acoustic wave sparsely activated localization microscopy (fast-awsalm): Ultrasound super-resolution using plane-wave activation of nanodroplets," IEEE Trans. Ultrason., Ferroelectr., Freq. Control, vol. 66, no. 6, pp. 1039-1046, 2019.

[28] G. P. Luke, A. S. Hannah, and S. Y. Emelianov, "Super-resolution ultrasound imaging in vivo with transient laser-activated nanodroplets," Nano Letters, vol. 16, pp. 2556-2559, 2016.

[29] H. Yoon, K. A. Hallam, C. Yoon, and S. Y. Emelianov, "Super-resolution imaging with ultrafast ultrasound imaging of optically triggered perfluorohexane nanodroplets," IEEE Trans. Ultrason., Ferroelectr., Freq. Control, vol. 65, no. 12, pp. 2277-2285, 2018.

[30] G. Zhang, S. Harput, S. Lin, K. Christensen-Jeffries, C. H. Leow, J. Brown, C. Dunsby, R. J. Eckersley, and M.-X. Tang, "Acoustic wave sparsely activated localization microscopy (awsalm): Super-resolution ultrasound imaging using acoustic activation and deactivation of nanodroplets," Applied Physics Letters, vol. 113, no. 1, p. 014101, 2018.

[31] E. Boni, L. Bassi, A. Dallai, F. Guidi, V. Meacci, A. Ramalli, S. Ricci, and P. Tortoli, "Ula-op 256: A 256-channel open scanner for development and real-time implementation of new ultrasound methods," IEEE Trans. Ultrason., Ferroelectr., Freq. Control, vol. 63, no. 10, pp. 14881495, 2016.

[32] E. Boni, L. Bassi, A. Dallai, V. Meacci, A. Ramalli, M. Scaringella, F. Guidi, S. Ricci, and P. Tortoli, "Architecture of an ultrasound system for continuous real-time high frame rate imaging," IEEE Trans. Ultrason., Ferroelectr., Freq. Control, vol. 64, no. 9, pp. 1276-1284, 2017. 